\title{
1 Identifying latent behavioral states in animal movement with M4, a non-parametric
}

\section{Bayesian method}

3

4 Joshua A Cullen ${ }^{1 *}$, Caroline L Poli ${ }^{2}$, Robert J Fletcher, Jr. ${ }^{3}$, Denis Valle ${ }^{1}$

5

$6{ }^{1}$ School of Forest Resources and Conservation, University of Florida, Gainesville, FL, USA

$7 \quad{ }^{2}$ School of Natural Resources and Environment, University of Florida, Gainesville, FL, USA

$8{ }^{3}$ Department of Wildlife Ecology and Conservation, University of Florida, Gainesville, FL, USA

9

$10{ }^{*}$ Corresponding Author: 136 Newins-Ziegler Hall, Gainesville, FL 32611. PO BOX 110410.

11 Email: joshcullen10@ gmail.com

12

13 Running headline: Flexible Bayesian estimation of behavior

14

15

16

17

18

19

20

21

22 Article type: Research article 


\section{Abstract}

24 1. Understanding animal movement often relies upon telemetry and biologging devices. These

25 data are frequently used to estimate latent behavioral states to help understand why animals

26 move across the landscape. While there are a variety of methods that make behavioral inferences

27 from biotelemetry data, some features of these methods (e.g., analysis of a single data stream,

28 use of parametric distributions) may limit their generality to reliably discriminate among

29 behavioral states.

31 2. To address some of the limitations of existing behavioral state estimation models, we

32 introduce a non-parametric Bayesian framework called the mixed-membership method for

33 movement (M4), which is available within the open-source bayesmove R package. This

34 framework can analyze multiple data streams (e.g., step length, turning angle, acceleration)

35 without relying on parametric distributions, which may capture complex behaviors more

36 successfully than current methods. We tested our Bayesian framework using simulated

37 trajectories and compared model performance against two segmentation methods (behavioral

38 change point analysis (BCPA) and segclust2d), one machine learning method (expectation-

39 maximization binary clustering $(\mathrm{EMbC})$ ), and one type of state-space model (hidden Markov

40 model (HMM)). We also illustrated this Bayesian framework using movements of juvenile snail

41 kites (Rostrhamus sociabilis) in Florida, USA.

42

43 3. The Bayesian framework estimated breakpoints more accurately than the other segmentation

44 methods for tracks of different lengths. Likewise, the Bayesian framework provided more

45 accurate estimates of behavior than the other state estimation methods when simulations were 
46 generated from less frequently considered distributions (e.g., truncated normal, beta, uniform).

47 Three behavioral states were estimated from snail kite movements, which were labeled as

48 'encamped', 'area-restricted search', and 'transit'. Changes in these behaviors over time were

49 associated with known dispersal events from the nest site, as well as movements to and from

50 possible breeding locations.

51

52 4. Our non-parametric Bayesian framework estimated behavioral states with comparable or

53 superior accuracy compared to the other methods when step lengths and turning angles of

54 simulations were generated from less frequently considered distributions. Since the most

55 appropriate parametric distributions may not be obvious a priori, methods (such as M4) that are

56 agnostic to the underlying distributions can provide powerful alternatives to address questions in

57 movement ecology.

58

59 Keywords: animal movement, Bayesian, behavioral state, biologging, movement ecology, non-

60 parametric, segmentation, telemetry 


\section{$61 \quad 1$. Introduction}

62

Our understanding of animal movement has advanced considerably in recent decades with the emergence of the field of movement ecology (Fraser et al. 2018; Joo, Picardi, et al. 2020), which focuses on understanding where animals go, what they are doing, and how they are influenced by their surrounding environment (Nathan et al. 2008). As telemetry and biologging devices continue to increase in their battery life, data resolution, and affordability (Hussey et al. 2015; Kays et al. 2015), statistical methods that can efficiently analyze these large datasets will become ever more important (Patterson et al. 2017; Potts et al. 2018). To fully understand animal movement, it is necessary to account for behavior since space and resource use are directly linked to an animal's internal state (Nathan et al. 2008; Gurarie et al. 2016).

Since the direct observation of animal behavior can be challenging in many situations, recorded tracks from biologging devices are increasingly used to infer potential behavior by estimating latent states. These latent states can be estimated from a variety of data streams (i.e., time series of variables) such as step lengths, turning angles, ambient temperature, and acceleration, among others (Edelhoff et al. 2016). State estimation is often performed using segmentation and clustering methods, as well as state-space models (SSMs). Segmentation methods partition tracks into segments by detecting shifts in the data stream(s), whereas clustering methods classify these segments (or the observations directly) into discrete states. Alternatively, SSMs estimate latent states per observation based on the transition probabilities among a given number of states (Edelhoff et al. 2016; Gurarie et al. 2016). While existing state estimation methods provide fast or powerful predictive capacity (Edelhoff et al. 2016; Patterson 
83 et al. 2017), they possess a number of limitations that can impact the inference made on

84 behavioral states.

For instance, segmentation methods commonly infer behavior using only a single data

86 stream such as persistence velocity or speed (Edelhoff et al. 2016; but see Patin et al. 2020). This

87 can be problematic when underlying behaviors are complex and not well represented by a single

88 metric alone. Additionally, many segmentation methods, clustering methods, and SSMs typically

89 estimate behavioral states by fitting the data streams to parametric probability distributions (e.g.,

90 Edelhoff et al. 2016; Patterson et al. 2017; Joo, Boone, et al. 2020), such as Gaussian, gamma, or

91 wrapped Cauchy distributions. When the structure in the data streams is not well captured by

92 parametric distributions, this can often result in overestimation of the true number of states when

93 information criteria are used due to model misspecification (Gurarie et al. 2016; Pohle et al.

94 2017). Furthermore, running SSMs and some clustering methods can be computationally costly:

95 model runtime can take minutes to days depending on the type of model, sample size, number of

96 estimated states, and computer hardware. This is further exacerbated when model selection (e.g.,

97 determining the likely number of groups by fitting models with different numbers of groups) and

98 multi-model inference are performed.

99 Given the limitations posed by existing state estimation methods, there is a need to

100 develop a framework that is based on as few parametric assumptions as possible while also being

101 fast and flexible. Here, we introduce a new two-stage modeling framework called the mixed-

102 membership method for movement (M4) that implements non-parametric Bayesian methods to:

103 1) segment multiple data streams into relatively homogeneous units of behaviors; and 2)

104 subsequently determine the likely number of behavioral states using a mixed-membership

105 method where segments can be comprised of more than one behavioral state. Latent behavioral 
106 states are estimated for entire track segments (as opposed to individual observations) since this

107 reflects our understanding that behavior is inherently autocorrelated, especially when

108 observations are sampled at short time intervals (Pohle et al. 2017; Potts et al. 2018).

109 Additionally, track segments are expected to be characterized by multiple states (Pohle et al.

110 2017; Patin et al. 2020). This M4 model framework is available within the open-source R

111 package bayesmove available on CRAN (Cullen and Valle 2021). In this article, we describe

112 the model structure and the Bayesian sampling methods used to estimate from the posterior

113 distribution. We then demonstrate that M4 can successfully recover breakpoints and behavioral

114 states based on simulated trajectories and compare our model's performance against two

115 common segmentation methods (behavioral change point analysis (BCPA), Gurarie et al. 2009;

116 segclust2d, Patin et al. 2020), one machine learning method (expectation-maximization binary

117 clustering (EMbC), Garriga et al. 2016), and one type of SSM, a hidden Markov model (HMM).

118 Finally, we illustrate our novel approach on the movements of an endangered raptor species, the 119 Everglade snail kite (Rostrhamus sociabilis), and interpret the results within the context of natal 120 and breeding dispersal events.

\section{2. Materials and Methods}

\section{$123 \quad 2.1$ Model Structure}

124 Most existing segmentation methods (e.g., BCPA, segclust2d, behavioral movement

125 segmentation), some machine learning methods (e.g., EMbC), and most SSMs (e.g., HMMs,

126 multistate random walks) experience one or more common limitations to behavioral state

127 estimation. These limitations include the reliance on parametric distributions, analysis of only a 
128 single data stream, as well as reliance on information criteria to determine the most likely

129 number of states.

\subsubsection{Discretization of data streams}

We address the problem of parametric distributions by providing an approach that relaxes

133 parametric assumptions through the discretization of data streams (Figs 1a, b, c). Although data

134 streams (e.g., step lengths and turning angles) are not typically discretized into bins, we expect

135 that this may lead to more robust estimates in the face of parametric distribution uncertainty.

136 This is because bins are estimated independently of one another and extreme values lose their

137 influence when added to the first or last bins with the rest of the data. Therefore, the

138 discretization of data streams is expected to increase model flexibility (Kitagawa 1987; John and 139 Langley 1995).

140 Selecting the number of bins and the binning method is relatively subjective and

141 therefore it is important that prior biological reasoning be used to inform these decisions. For

142 example, discretization methods may include the use of equal bin widths or quantiles. However,

143 the number of bins should be sufficient to characterize the shape of the density distribution.

144 These assumptions during the discretization process are not unlike assumptions made for HMMs

145 when selecting probability distributions to fit data streams, but require practitioners to make

146 more decisions up front. Based on a sensitivity analysis of binning methods used on a right-

147 skewed data stream (i.e., step lengths), the use of quantiles resulted in greater discrimination of

148 behavioral states than bins of equal widths (Appendix S1). However, data streams with circular

149 distributions (e.g., turning angles) will likely be more interpretable when using bins of equal 
150 widths. At a minimum, discretized values for each data stream and the associated track IDs are

151 required to begin analyzing the data.

\subsubsection{Segmentation}

Similar to other segmentation methods, our model aims to divide tracks into segments by estimating a set of breakpoints. Through the use of joint probabilities within a Bayesian framework, we circumvent the limitation of analyzing only a single data stream. Moreover, our model estimates the location and number of unknown breakpoints by implementing a Gibbs

158 sampler within a reversible-jump Markov chain Monte Carlo (RJMCMC) algorithm. RJMCMC

159 is a trans-dimensional algorithm that serves as a model-based approach to model selection by

160 providing simultaneous inference on parameter values given a particular model, as well as model

161 space (i.e., the collection of all possible models) (Green 1995). In particular, we use a birth-death

162 RJMCMC that allows the addition (i.e., birth), removal (i.e., death), or swap of proposed

163 breakpoints where model parameters are updated from the known posterior distribution using a

164 Gibbs sampler (see Appendix S2 for more details). We adopt this approach to perform

165 unsupervised segmentation on each individual trajectory. In our framework, each potential model $166 M_{k}$ is characterized by a set of $P$ breakpoints $\left\{b_{1 k}, \ldots, b_{P k}\right\}$, where $k$ is the model number. Each

167 breakpoint is restricted to being an integer between 2 and $T_{i-1}$ across all observations, where $T_{i}$

168 is the total number of observations for individual $i$. Given a particular model $M_{k}$, its breakpoints

169 define track segments. We assume that for any given track segment $c$ :

$$
x_{i t j} \sim \operatorname{Categorical}\left(\boldsymbol{\theta}_{\boldsymbol{c} j}\right)
$$


173 where $x_{i t j}$ is the bin label for individual $i$ at time $t$ for data stream $j$ and $\boldsymbol{\theta}_{\boldsymbol{c} j}$ is a vector of

174 probabilities that sum to one. The vector $\boldsymbol{\theta}_{\boldsymbol{c} \boldsymbol{j}}$ indicates the probability that observations within 175 segment $c$ are assigned to one of $L$ bins. Overall, the model is seeking breakpoints that define 176 relatively homogeneous track segments. The use of a categorical distribution to characterize

177 track segments (as opposed to continuous distributions) is what makes this framework non178 parametric. Our prior is given by:

$$
\boldsymbol{\theta}_{\boldsymbol{c j}} \sim \operatorname{Dirichlet}(\alpha)
$$

182 where $\alpha>0$ is equal across all bins. We integrate over the latent parameter $\boldsymbol{\theta}_{\boldsymbol{c} j}$ to enable the 183 algorithm to visit multiple models and increase computational efficiency. Since there are no

184 longer any remaining parameters on which to assess model convergence, we do so by evaluating trace-plots of the log marginal likelihood (Denison et al. 2002). Details for the derivation of the full conditional distributions can be found in Appendix S3.

Since the posterior distribution of models $M_{k}$ can vary greatly in the number and position

188 of breakpoints, but only a single set of breakpoints can be used to define track segments, we

189 select the Maximum a Posteriori (MAP) estimate (i.e., the breakpoints of the model with the 190 greatest log marginal likelihood) (Fig. 1d). Although the MAP estimate does not account for

191 uncertainty in breakpoint number and position, it appears to be in good agreement with estimates

192 from the entire posterior distribution as described in Appendix S4. These MAP breakpoints are

193 then used to define segments per individual track, which are subsequently clustered into latent

194 behavioral states by a mixed-membership model. 


\subsubsection{Mixed-membership clustering}

Although most existing state estimation methods assign a single discrete state to

Dirichlet Allocation (LDA), a mixed-membership clustering method, can be used to classify each track segment as a mixture of multiple states (Valle et al. 2014; Hudon et al. 2021). For example,

206 where each state corresponds to a distribution of discretized data streams. To do so, the model

207 estimates the probability of observations from each track segment (rows) belonging to each

208 latent state (columns) in matrix $\Theta$ (Fig. 1f). Additionally, the model characterizes the latent states

209 (rows) with the probability of observations belonging to each bin per discretized data stream

210 (columns) in matrix $\boldsymbol{\Phi}$ (Fig. 1e). The track segments from all individual animals are analyzed

211 together since we assume that there is a common set of behaviors exhibited across the

212 population. Although there may be some individual heterogeneity in movement patterns, the

213 pooling of all individuals ensures that behavioral states are directly comparable and improves the

214 inference on individuals with fewer observations (Jonsen 2016). In this model, we assume that:

$$
y_{i j c t} \mid\left\{z_{i j c t}=k\right\} \sim \text { Categorical }\left(\boldsymbol{\phi}_{k j}\right)
$$


218 where $y_{i j c t}$ denotes the bin for time $t$ of track segment $c$ from data stream $j$ for individual $i$.

219 Additionally, $z_{i j c t}$ is the latent behavioral state membership associated with $y_{i j c t}$ and $\boldsymbol{\phi}_{\boldsymbol{k} j}$ is a

220 vector of probabilities for each behavior and data stream. Notice that $z_{i j c t}$ influences the

221 distribution for $y_{i j c t}$ by determining the subscript $k$ for the vector $\boldsymbol{\phi}_{\boldsymbol{k} \boldsymbol{j}}$. We assume that the latent

222 state membership is given by:

$$
z_{i j c t} \sim \text { Categorical }\left(\boldsymbol{\theta}_{i c}\right)
$$

where $\boldsymbol{\theta}_{\boldsymbol{i c}}$ is a vector of probabilities of size $K$ (i.e., the number of clusters or states) that sum to one and indicates the likelihood of assigning an observation at time $t$ of track segment $c$ for comprised of multiple states. For our priors, we assume that:

$$
\boldsymbol{\phi}_{k j} \sim \operatorname{Dirichlet}(\alpha)
$$

$$
\boldsymbol{\theta}_{\text {ic }} \sim T S B(\gamma)
$$

234 where $\operatorname{TS} B(\gamma)$ represents the truncated stick-breaking prior from Bayesian non-parametrics. This

235 prior is given by:

$$
\theta_{i c k}=V_{i c k} \prod_{p=1}^{k-1}\left(1-V_{i c p}\right)
$$

$$
V_{i c k} \sim \operatorname{Beta}(1, \gamma)
$$


239 where $V_{K}=1$ and $\gamma>0$. By setting $0<\gamma<1$, we can shrink the probability of assigning state

$240 k$ to track segment $c$ (i.e., $\theta_{i c k}$ ) to approximately zero as $k$ approaches $K$. As a result, fewer and

241 fewer observations will be assigned to states with large values of $k$, enabling the model to

242 identify the most likely number of behavioral states (Valle et al. In Review; Valle et al. 2017).

243 This is an improvement on existing state estimation methods in the sense that our model only

244 needs to be run once, whereas several other common methods (e.g., HMMs, segclust2d, and

245 other clustering methods) are typically run multiple times with varying numbers of behavioral

246 states to then determine the best model via information criteria (e.g., AIC or BIC).

247 This LDA model is fitted using a Gibbs sampler and a complete description of the full

248 conditional distribution can be found in Appendix S5. Similar to the segmentation model,

249 convergence was assessed by inspecting trace-plots of the log-likelihood. The posterior mean for

250 all $\boldsymbol{\theta}_{i c}$ was then used to identify the most likely number of behaviors. The estimated state-

251 dependent distributions for each data stream (from $\boldsymbol{\phi}_{\boldsymbol{k}}$ ) were evaluated and used to corroborate

252 the findings based on the posterior means from all $\boldsymbol{\theta}_{\boldsymbol{i c}}$ 's by determining whether the distributions

253 were biologically relevant (Figs 1e, f, g). This combination of results provides a straightforward

254 approach to selecting the most likely number of behavioral states. A list of the primary functions

255 to analyze data using the M4 framework within the bayesmove R package is included in

256 Appendix S6. 
1) Discretize data streams (i.e., bin the data)

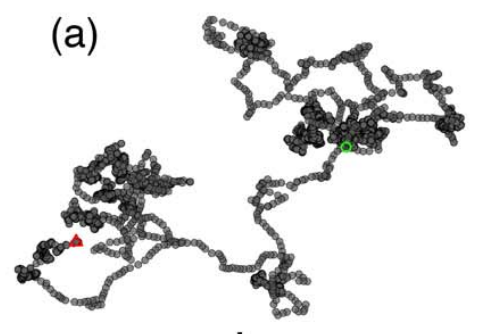

2) Segment the track (create breakpoints)

3) LDA clustering and behavior estimation

\section{4) Annotate track}

(b)

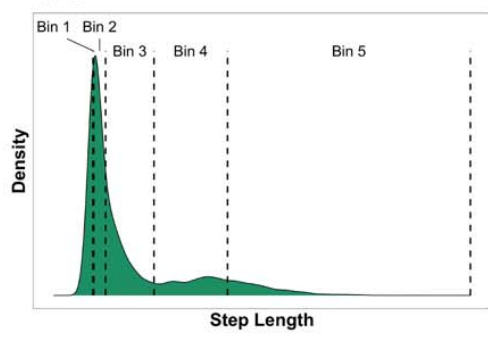

(d)
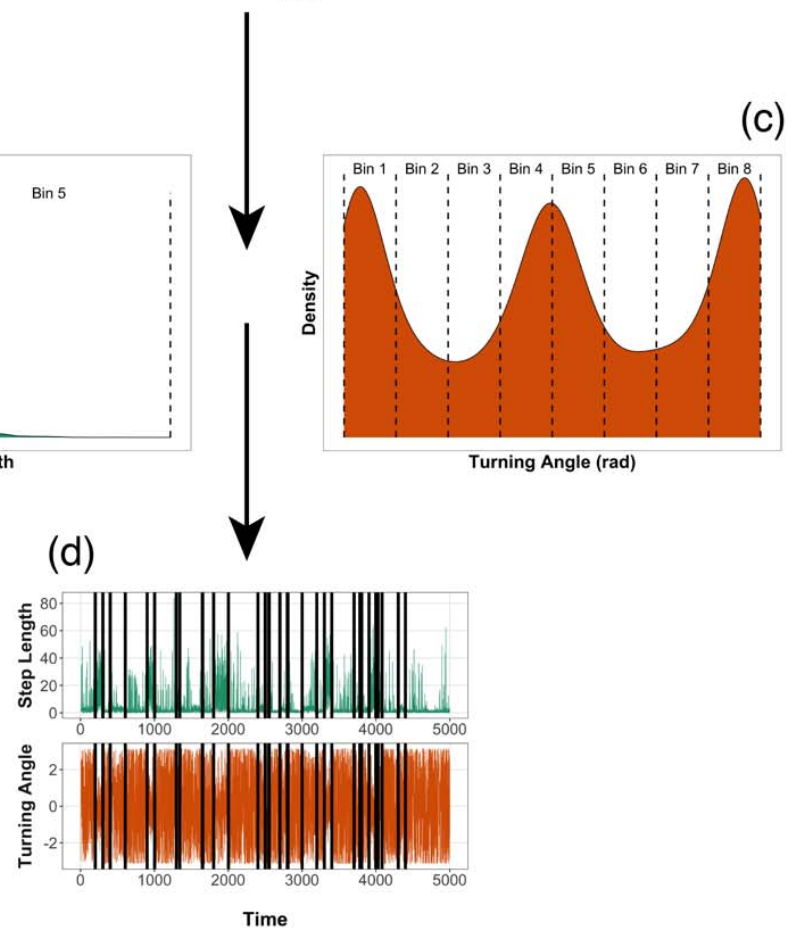

(e)

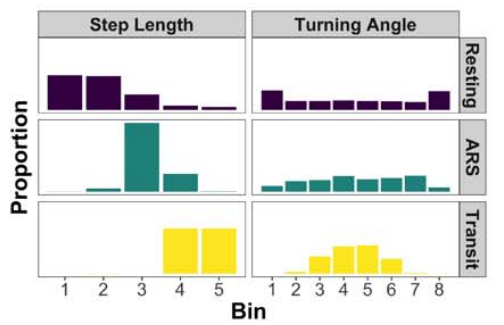

$\downarrow$

(f)
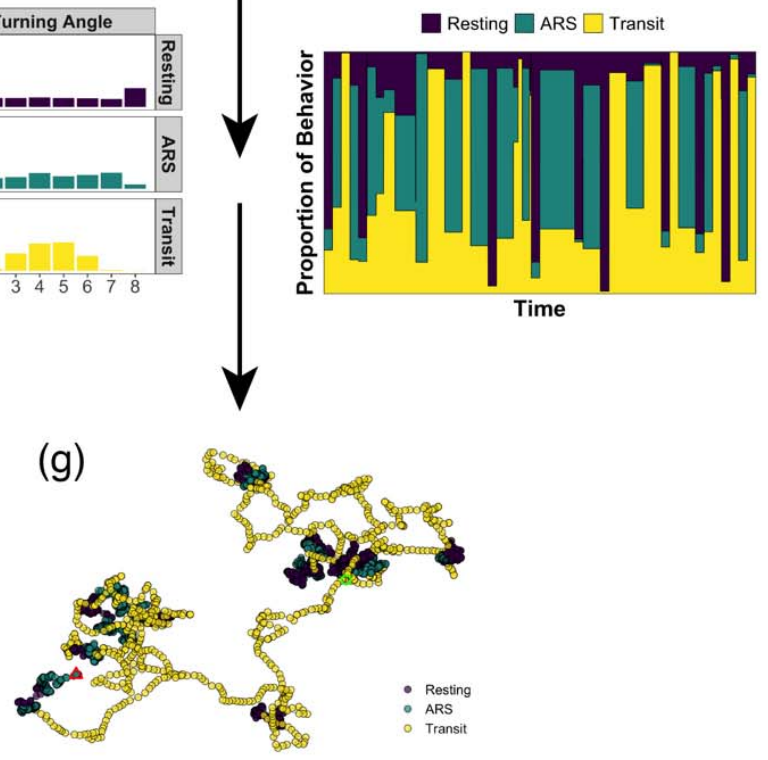
Figure 1 General workflow to analyze animal trajectories from telemetry data using M4. Steps from this analysis include: (a)

starting with a track of coordinates that are used to calculate step lengths and turning angles, (b) discretizing step lengths and

(c) turning angles into sets of bins, (d) performing segmentation on the joint time series of step lengths and turning angles, (e)

cluster the track segments into behavioral states by Latent Dirichlet Allocation (LDA) (matrix $\Phi$ ) and (f) evaluate time series of

\subsection{Simulation Study}

We assessed the performance of M4 compared to other methods via simulations. We first evaluated the ability of our track segmentation method to detect true breakpoints and compared

270 its results to those obtained by two segmentation methods (i.e., BCPA and segclust2d). We then

271 evaluated the ability of our clustering method to estimate the true number of behavioral states

272 and to properly assign behavior proportions to track segments. For this component, we compared

273 the results of our model to those obtained by a HMM (McClintock and Michelot 2018) and two

274 additional clustering methods (i.e., segclust2d and EMbC).

\subsubsection{Generating simulated trajectories}

We generated multiple three-state trajectories from a correlated random walk at regular

278 time intervals, where five tracks were simulated at each of four durations $(1000,5000,10000$,

27950000 observations), resulting in a total of 20 tracks. Each track was comprised of 10, 50, 100, or

280500 segments that each included 100 observations. Each of these segments included a dominant

281 behavioral state ( $80 \%$ of observations), which was randomly assigned to each segment. The three

282 behavioral states were parameterized to represent (1) little to no movement ('encamped'), (2)

283 slow and tortuous movement ('area-restricted search' or ARS), as well as (3) fast and directed 
284 movement ('transit'). To assess model performance on tracks generated from different types of

285 distributions, we generated two sets of tracks with 20 simulations in each. In the first set of

286 simulations, step lengths for each behavior were drawn from a truncated normal distribution and

287 turning angles were drawn from either a beta, uniform, or truncated normal distribution

288 (hereafter referred to as 'uncommon distributions') (Fig. 2a). For the second set of simulations,

289 step lengths for each behavioral state were generated from a gamma distribution and turning

290 angles were drawn from a wrapped Cauchy distribution (hereafter referred to as 'common

291 distributions') (Fig. 2b). Both sets of simulations were designed to generally resemble each other

292 in their step length and turning angle distributions. 
bioRxiv preprint doi: https://doi.org/10.1101/2020.11.05.369702; this version posted June 9, 2021. The copyright holder for this preprint (which was not certified by peer review) is the author/funder, who has granted bioRxiv a license to display the preprint in perpetuity. It is made available under aCC-BY 4.0 International license.
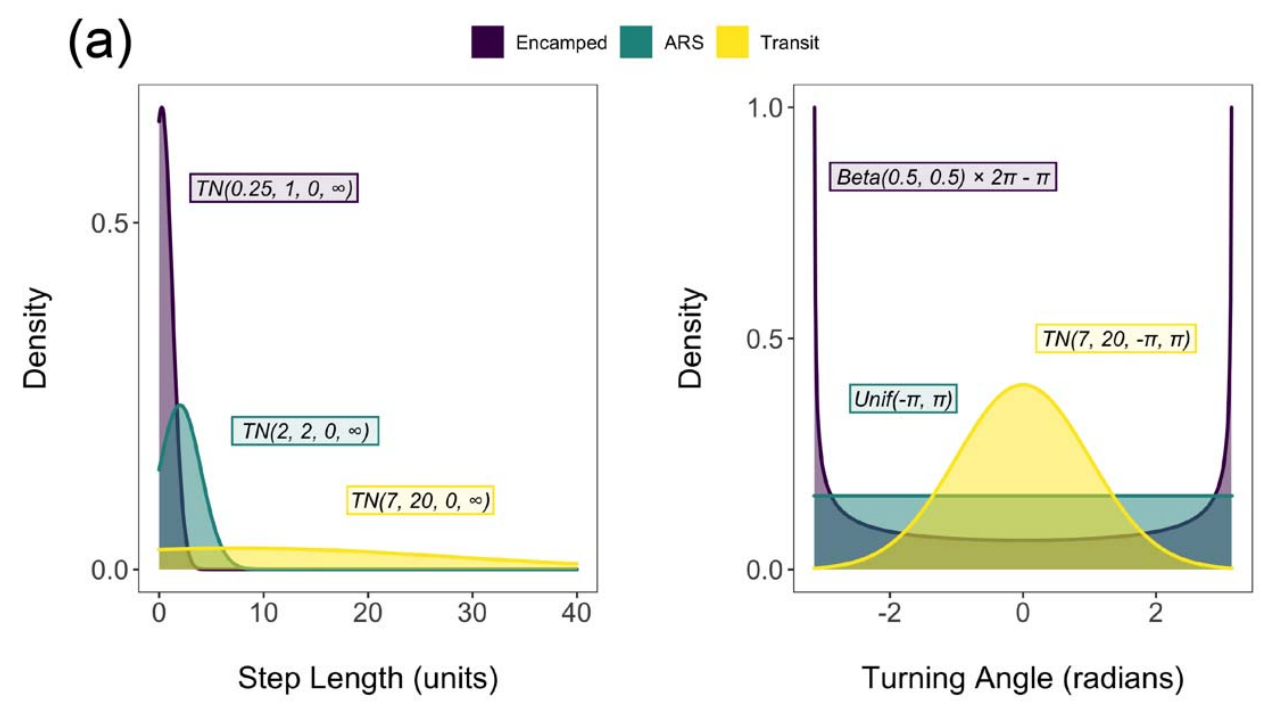

(b)
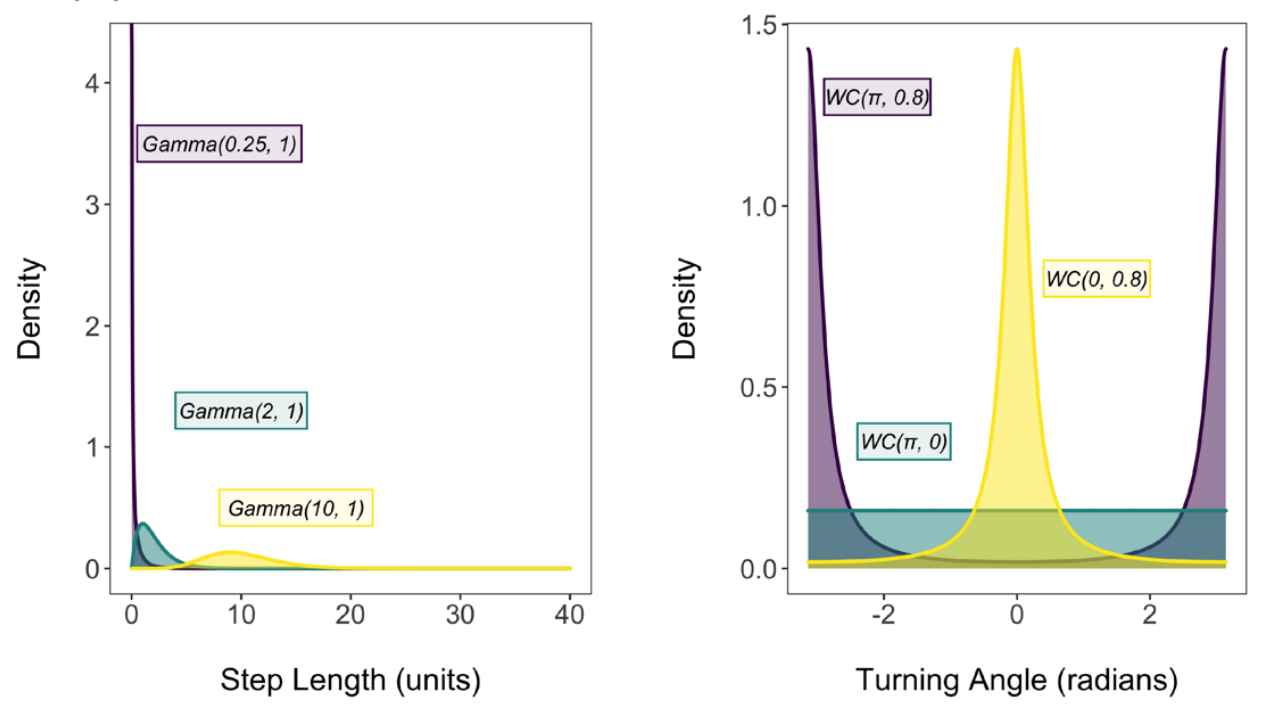

295 Figure 2 Uncommon (a) and common (b) distributions used to generate step lengths and turning angles for each simulated state.

296 (a) Step lengths are generated from a truncated normal (TN) and turning angles are generated from beta, uniform, and truncated

297 normal distributions. TN $(a, b, c, d)$ denotes a truncated normal distribution with mean a, standard deviation $b$, lower bound $c$, and

298 upper bound d. (b) Step lengths are generated from a gamma distribution and turning angles are generated from a wrapped

299 Cauchy (WC) distribution. 
Step lengths and turning angles were the data streams used to make inference on latent

303 behavioral states. Step lengths were separated into five bins using the $25^{\text {th }}, 50^{\text {th }}, 75^{\text {th }}, 90^{\text {th }}$, and

$304100^{\text {th }}$ quantiles as upper limits. Quantiles were used to discretize highly right-skewed step

305 lengths as suggested by our sensitivity analysis (Appendix S1). Turning angles were discretized

306 into eight bins from $-\pi$ to $\pi$ using equal widths $\left(\frac{\pi}{4}\right)$ since the distribution of this variable was

307 relatively balanced and bounded by lower and upper limits.

308 Each simulated track was analyzed by the M4 segmentation model using a vague prior

309 where the hyperparameter $\alpha$ was set to 1 . Trace-plots of the log marginal likelihood indicated

310 that the model reached convergence for each simulation, where 40,000 MCMC iterations were

311 used for all but the longest tracks, which used 60,000 iterations (Appendix S7). We then assessed

312 how well our model identified the true breakpoints for each simulation, where a threshold of \pm 10

313 observations was used to distinguish an accurate from an inaccurate estimated breakpoint. If no

314 breakpoints were estimated within \pm 30 observations of a true breakpoint, then the model was

315 considered to have 'missed' that breakpoint. Other thresholds were tested and all resulted in the

316 same relative pattern of accuracy (Appendix S1).

Estimated track segments were used as input for the LDA model of M4, which was run

318 using $1000 \mathrm{MCMC}$ iterations, a burn-in of 500 iterations, and vague priors where

319 hyperparameters were set to $\gamma=0.1$ and $\alpha=0.1$. We set the maximum number of behavioral

320 states to seven since this was expected to include the true number of states, estimated via the

321 truncated stick-breaking prior of the LDA. Trace-plots of the log-likelihood indicated that the

322 model reached convergence for each simulation (Appendix S7). The true number of states was

323 estimated by calculating the arithmetic mean of behavior proportions across all track segments

324 and selecting the set of states that together represented $\geq 90 \%$ of all observations on average. 
325 Additionally, state-dependent distributions of step lengths and turning angles were inspected so

326 that we only selected states that were also biologically interpretable. Since the LDA treats track

327 segments as a combination of behavioral states, proportions of each state were estimated per

328 track segment. Accuracy of state estimates were evaluated by two methods: 1) we calculated the

329 percentage of observations where the dominant behavior of each track segment was accurately

330 classified, and 2) we calculated the root mean square error (RMSE) of the estimated behavior

331 proportions compared to the true behavior proportions over all states and track segments.

\subsubsection{Method comparison}

We compared the performance of M4 on the simulated trajectories against BCPA, EMbC,

335 HMM, and segclust2d (see Appendix S8 for details regarding model properties and assumptions). All models were run using a $2.6 \mathrm{GHz}$ i7 CPU with $16 \mathrm{~GB}$ RAM.

\subsubsection{Segmentation models}

The BCPA model performed segmentation based on persistence velocity (PV), which is a

340 combination of velocity $(V)$ and turning angle $(\theta)$ (i.e., $P V=V \cos (\theta)$ ), using the $\mathrm{R}$ package

341 bcpa v1.1 (Gurarie 2014). Parameters for BCPA were tuned to provide a close approximation

342 of the true number and location of simulated breakpoints with window size set to 80 , sensitivity

343 set to 2, and clusterwidth set to 30. Breakpoint accuracy was evaluated using the same method as

344 for M4.

The segclust $2 \mathrm{~d}$ model performed segmentation on step lengths and the absolute value of

346 turning angles using the R package segclust2d v0.2.0 (Patin et al. 2019). This method

347 models each data stream using a Gaussian distribution, so the absolute value of turning angles 
was used to accommodate this unimodal assumption. Tuning parameters were chosen within the bounds of the simulated tracks, such that the maximum number of segments was set to $1.5 \times s$

350 where $s$ is the true number of segments, the minimum observations per segment was set to 50, 351 and the number of potential clusters (i.e., states) ranged from 2 to 4 . Since the model was still

352 analyzing the longest simulations (50000 observations) after two days, these tracks were omitted

353 from the reported results for segclust2d. Breakpoint accuracy was assessed in the same manner

354 as for M4.

\subsubsection{Clustering models}

The EMbC model was fitted to step lengths and the absolute value of turning angles

358 using the R package EMbC v2.0.3 (Garriga et al. 2019). The absolute value of turning angles was

359 used to achieve better discrimination among states given the use of a unimodal distribution like

360 the Gaussian distribution. This model uses binary clustering to partition each of $n$ data streams

361 into a 'low' and 'high' class, resulting in a total of $2^{\mathrm{n}}$ possible states. For our analysis, this

362 resulted in four states estimated from a bivariate Gaussian distribution. To make these results

363 comparable to the other models, both states with 'high' step lengths (and 'low' or 'high' turning

364 angles) were merged into a single state to produce three states overall. State classification

365 accuracy was assessed at the segment-level so that results were directly comparable with the

366 Bayesian M4 model. This was achieved by using true breakpoints to segment the time series of

367 states estimated by the EMbC model and then calculating the proportion of these behaviors

368 within each track segment. Additionally, the resulting state-dependent distributions of step

369 lengths and turning angles were discretized using the bin limits defined for M4 to compare the 
370 accuracy of distribution shapes. Accuracy was measured by RMSE across bins of all states and

371 data streams per simulation.

372 A discrete-time HMM was also fitted to each of the simulated trajectories using the R

373 package momentuHMM v1.5 (McClintock and Michelot 2018). Step lengths were modeled using

374 a gamma distribution and turning angles were assumed to arise from a wrapped Cauchy

375 distribution. The HMMs for each simulation were run using a range of 2 to 4 possible behavioral

376 states $(K)$ and each $K$-state model was run 30 times using different starting values to increase the

377 chance of finding the global (as opposed to local) maximum of the likelihood. The selection of

378 "good" starting values is critical since it can affect computation time and the ability of the model

379 to identify the global maximum of the likelihood (Michelot et al. 2016; McClintock and

380 Michelot 2018). The most likely number of states was selected using a combination of AIC and

381 BIC, where the model with the lowest value was considered to be most likely. However, if the

382 difference in $\mathrm{AIC}$ or $\mathrm{BIC}(\triangle \mathrm{AIC} / \mathrm{BIC})$ of the next best model was $<10$ (Burnham and Anderson

383 2002), the more parsimonious model was chosen. Behavior classification accuracy was assessed

384 in the same manner as for EMbC.

385 The segclust $2 \mathrm{~d}$ model clustered segments previously estimated by this method into $K$

386 states. The number of likely states was estimated using BIC in the same manner as for HMM.

387 The likely number of states (and associated breakpoints) were used to assign behavioral states to

388 track segments, which were then compared to the other methods using the proportion of each

389 state per estimated segment (which were all either 0 or 1). Additionally, the accuracy of the state-

390 dependent distributions were evaluated in the same manner as for EMbC and HMM. 


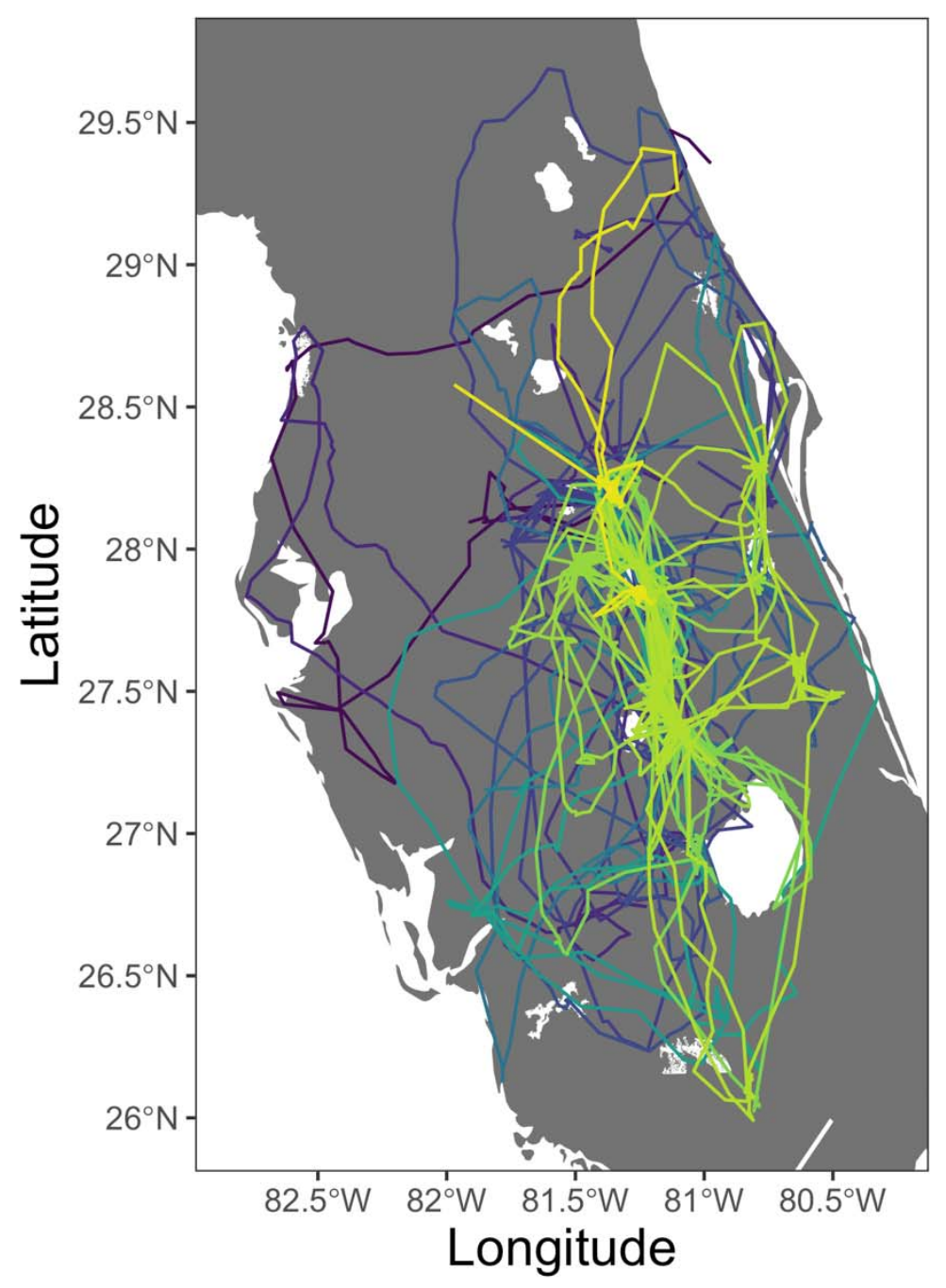

\subsection{Snail kite case study}

As part of a larger investigation on the effects of wetland management on wildlife, solar-

396 powered GPS-GSM transmitters (Ecotone Telemetry) were attached to juvenile snail kites

$397 \quad(n=26)$ prior to fledging at Lakes Tohopekaliga, East Tohopekaliga, and Kissimmee in central

398 Florida during 2018 and 2019. Subsequent movement of each individual resulted in a total of 
40,720 observations (Fig. 3). Locations were collected once per hour only during daylight at an

400

401

402

403

404

405

406

407

408

409

410

411

412

413

414

415

416

417

418

419

420

accuracy of $\pm 30 \mathrm{~m}$. As a result of the programmed duty cycle and time periods where GPS tags

failed to transmit data, track time intervals were irregular. To ensure comparable step lengths and turning angles, we filtered our data to the most common time interval (i.e., $1 \mathrm{~h}$ ). We chose to omit all other observations since imputation procedures for long time gaps would increase the number of artificial data and the use of linear interpolation would artificially inflate the number of turning angles at zero radians.

Step lengths and turning angles were used to estimate latent behavioral states. As was performed on the simulated tracks, step lengths for the empirical data were discretized into five bins using the $25^{\text {th }}, 50^{\text {th }}, 75^{\text {th }}, 90^{\text {th }}$, and $100^{\text {th }}$ quantiles as upper limits. This resulted in bin limits at $0.00,0.03,0.09,0.32,1.63$, and $72.56 \mathrm{~km}$. Turning angles were discretized into eight bins from $-\pi$ to $\pi$ using equal bin widths, resulting in bin limits at $-\pi,-\frac{3 \pi}{4},-\frac{\pi}{2},-\frac{\pi}{4}, 0, \frac{\pi}{4}, \frac{\pi}{2}, \frac{3 \pi}{4}, \pi$.

Step lengths and turning angles for each of the 26 snail kites were analyzed by the M4 segmentation model using 80,000 iterations, a burn-in of 40,000 iterations, and hyperparameter $\alpha=1$. The MAP estimates of breakpoints for each snail kite were used to define track segments per individual. Subsequently, track segments were analyzed across all individuals via LDA to estimate the most likely number of states, to define state-dependent distributions, and to estimate the proportion of each state that characterized each track segment. This was performed using 1000 MCMC iterations with a burn-in of 500 iterations and vague priors were used with hyperparameters set at $\gamma=0.1$ and $\alpha=0.1$ for a maximum possible number of seven states. Trace-plots of the segmentation and LDA models from M4 indicated that all had reached convergence (Appendix S7). Proportions of behavioral states were evaluated over time in 
421 relation to emigration from natal sites and peak breeding season of snail kites in Florida (March

4221 - June 30; Reichert et al. 2020) to discern any patterns associated with these events.

423

\section{3. Results}

4253.1 Segmentation model comparison

426 The M4 segmentation model successfully recovered breakpoints from the simulations and 427 outperformed both BCPA and segclust2d. Among the three methods, the segclust2d model took 428 much longer to run (0.46 to $418 \mathrm{~min}$ ) compared to M4 (1.98 to $227 \mathrm{~min}$ ) and BCPA models

429 (0.25 to $21 \mathrm{~min}$ ), particularly for longer tracks (Figs. 4a, 5a). While all three models exhibited

430 similar accuracy on the shortest simulations, M4 was much more accurate on all larger

431 simulations. For these large simulations, accuracy of the M4 segmentation model was $>80 \%$ on

432 average when simulations were generated from uncommon distributions and $>90 \%$ on average

433 when generated from common distributions (Figs. 4b, 5b, 6a). Additionally, M4 missed the

434 lowest proportion of true breakpoints (uncommon: 21\%; common: $0.3 \%$ ) compared to BCPA

435 (uncommon: 67\%; common: 66\%) and segclust2d (uncommon: 26\%; common: 30\%) across

436 simulations of all analyzed track lengths. 
bioRxiv preprint doi: https://doi.org/10.1101/2020.11.05.369702; this version posted June 9, 2021. The copyright holder for this preprint (which was not certified by peer review) is the author/funder, who has granted bioRxiv a license to display the preprint in perpetuity. It is made available under aCC-BY 4.0 International license.

$\mathrm{M} 4 \div \mathrm{HMM} \div$ Segclust2d $\div \mathrm{EMbC} \div \mathrm{BCPA}$

(a)

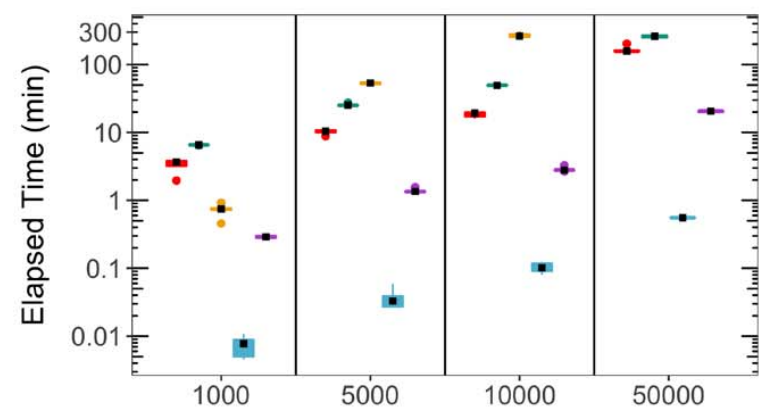

(c)

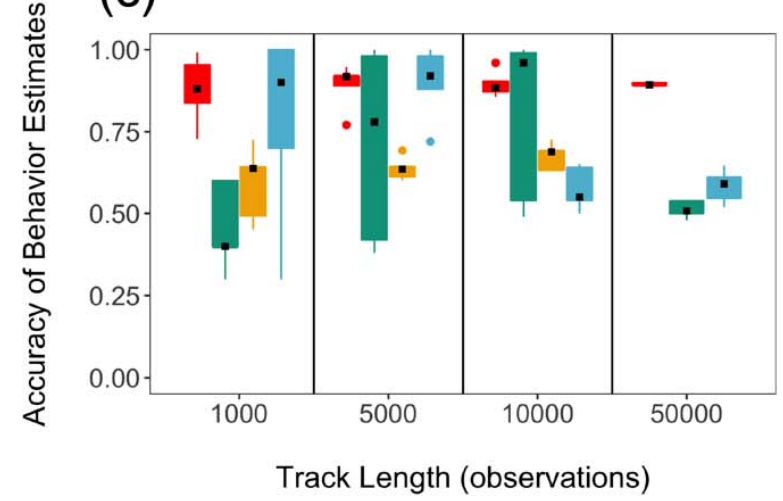

(b)

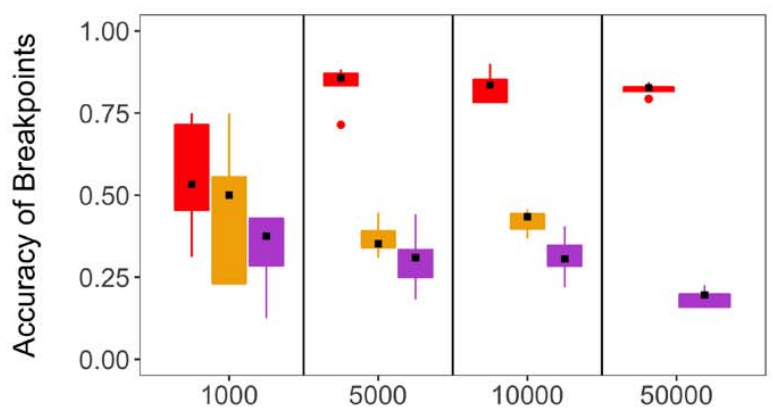

(d)

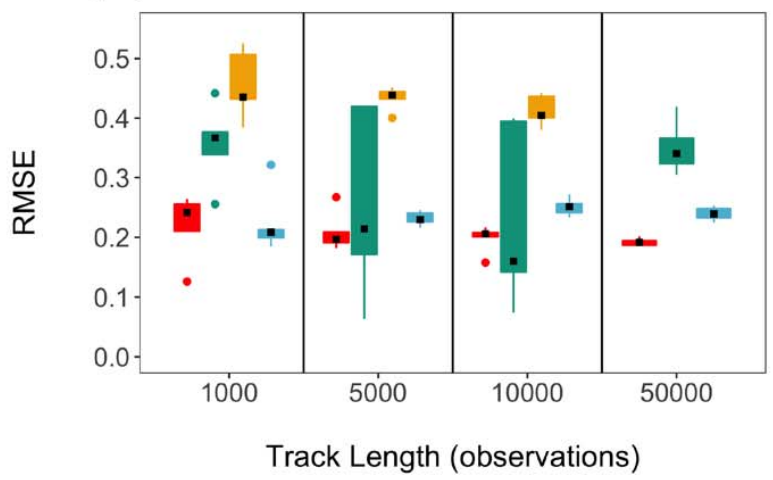

Figure 4 Comparison of the performance of different methods on tracks simulated from uncommon distributions, where black points indicate the median for each boxplot. (a) The elapsed time to analyze each of the simulations is shown for the different

441 methods on a logarithmic scale, where the measure for M4 reflects the sum of elapsed times from both the segmentation and

442 LDA models. (b) Accuracy of breakpoint estimates are compared among the M4, segclust2d, and BCPA models. (c) Accuracy of

443 the estimates for the dominant behavior of each track segment is shown for the M4, HMM, segclust2d, and EMbC models. (d)

444 Accuracy of behavior proportion estimates per track segment are compared among the M4, HMM, segclust2d, and EMbC 
(a)

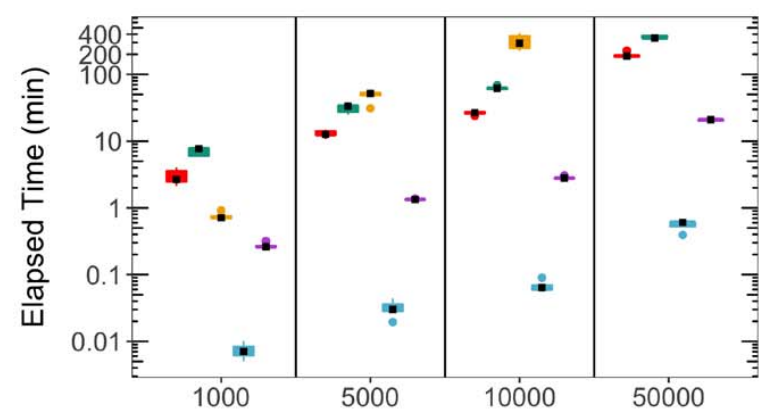

(c)

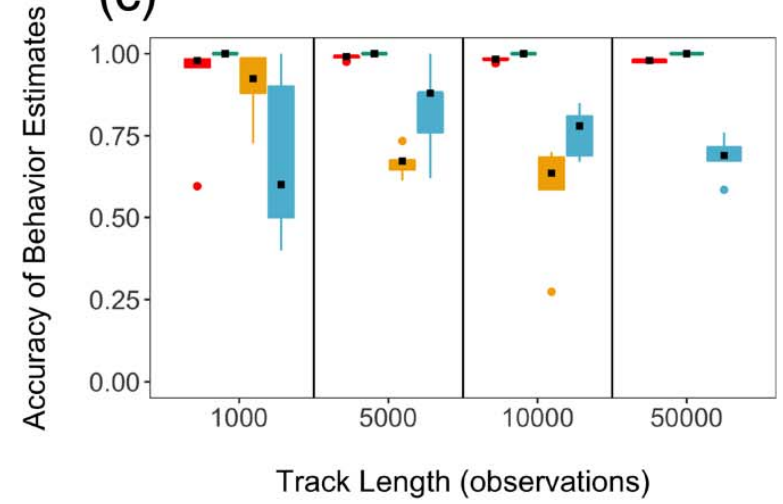

(b)

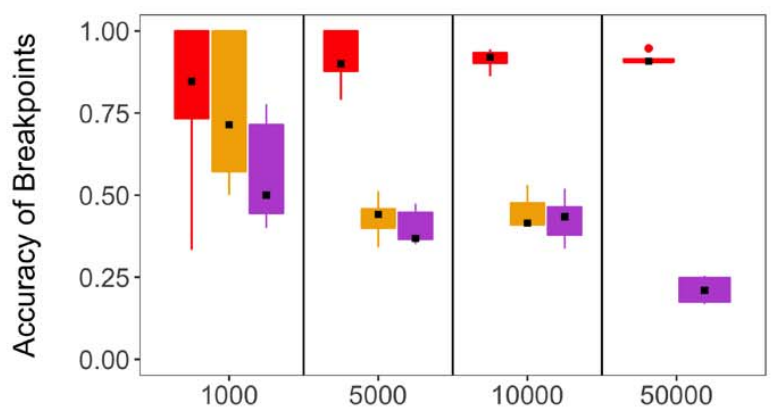

(d)

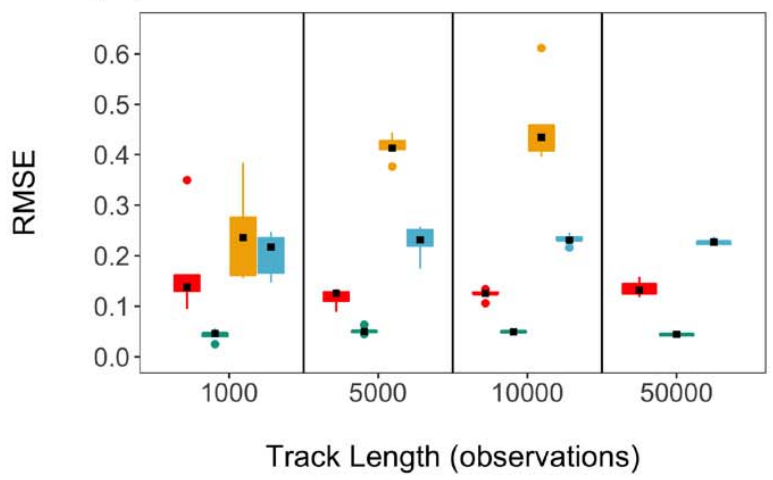

Figure 5 Comparison of the performance of different methods on tracks simulated from common distributions, where black

448 points indicate the median for each boxplot. (a) The elapsed time to analyze each of the simulations is shown for the different

449 methods on a logarithmic scale, where the measure for $M 4$ reflects the sum of elapsed times from both the segmentation and

450 LDA models. (b) Accuracy of breakpoint estimates are compared among the M4, segclust2d, and BCPA models. (c) Accuracy of

451 the estimates for the dominant behavior of each track segment is shown for the M4, HMM, segclust2d, and EMbC models. (d)

452 Accuracy of behavior proportion estimates per track segment are compared among the M4, HMM, segclust2d, and EMbC

453 methods.

\section{$454 \quad 3.2$ Clustering model comparison}

455 When estimating the true number of states on both sets of simulations, M4 correctly

456 determined the number of true states more frequently than the other methods and exhibited

457 greater computational efficiency over all other clustering methods besides EMbC. The Bayesian 
458 LDA model took $2-23$ s to run, highlighting the computational efficiency of this particular

459 model. When added to the duration of the segmentation model, the proposed method ran faster

460 than the HMM and segclust2d at all track lengths despite these models being fitted with only two

461 to four states whereas our method allows for up to seven states (Figs. 4a, 5a). However, the time

462 to run each EMbC model increased very little with increases in track length, but also

463 automatically assumed four states were present. The LDA model from M4 correctly suggested

464 three states as most likely for 18 of the 20 simulations generated from uncommon distributions

465 and 19 of 20 simulations generated from common distributions (Fig. 6b, Appendix S9). By

466 comparison, the HMM suggested (via AIC and BIC) that three states were most likely in 17 and

46716 of the 20 analyzed simulations generated by uncommon and common distributions,

468 respectively. The segclust $2 d$ model suggested that three states were most likely in only 6 and 5

469 of the 15 analyzed simulations generated from uncommon and common distributions,

470 respectively, based on BIC.

471

To enable direct comparisons among all four models that estimated behavioral states, we

472 assumed three states were most likely for all 20 simulations when calculating model accuracy.

473 Using this assumption, we find that M4 demonstrated high accuracy in behavioral state

474 estimation for both sets of simulations, often equivalent or superior to the other clustering

475 methods. When analyzing simulations generated from uncommon distributions, mean accuracy

476 of M4 to classify the dominant state within each segment was greater than that of the HMM and

477 segclust2d models at all track lengths (Fig. 4c). However, mean accuracy of the EMbC model

478 was slightly greater than M4 on this set of simulations at a track length of 5000 observations.

479 When analyzing simulations generated from common distributions, mean accuracy of M4 was

480 slightly below that of the HMM, but greater than the mean accuracy of the EMbC and segclust2d 
481 models at all track lengths (Fig. 5c). Additionally, accuracy measures displayed little variability

482 in M4 across tracks of different lengths and on each set of simulations, highlighting the increased

483 stability of this framework. Similar to the pattern found for estimates of dominant behavioral

484 states, accuracy of behavioral state proportions were higher in M4 for all but the HMM on

485 simulations generated from common distributions, as denoted by low RMSE (Figs. 4d, 5d, 6c).

(a)
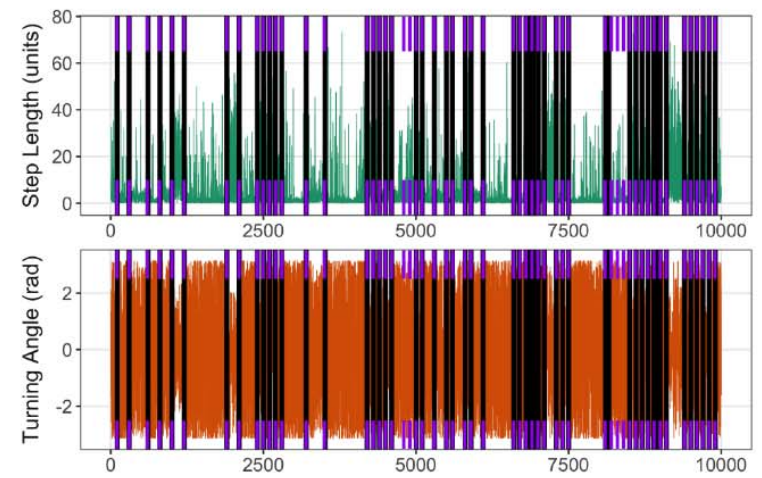

(c) (b)

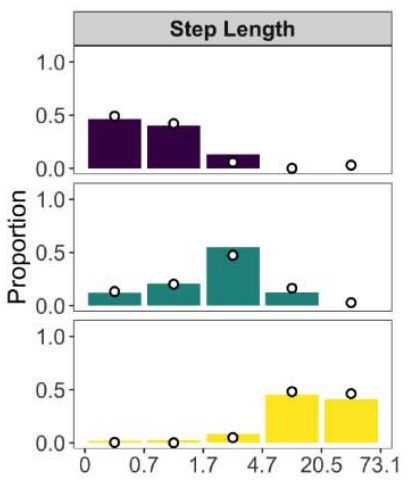

Bin

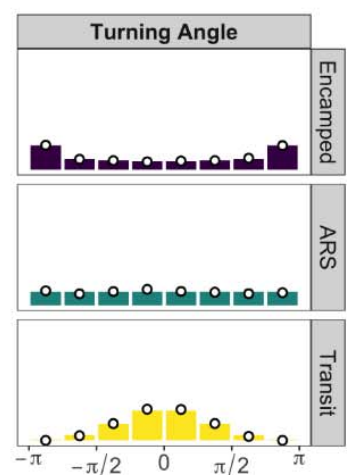

Bin

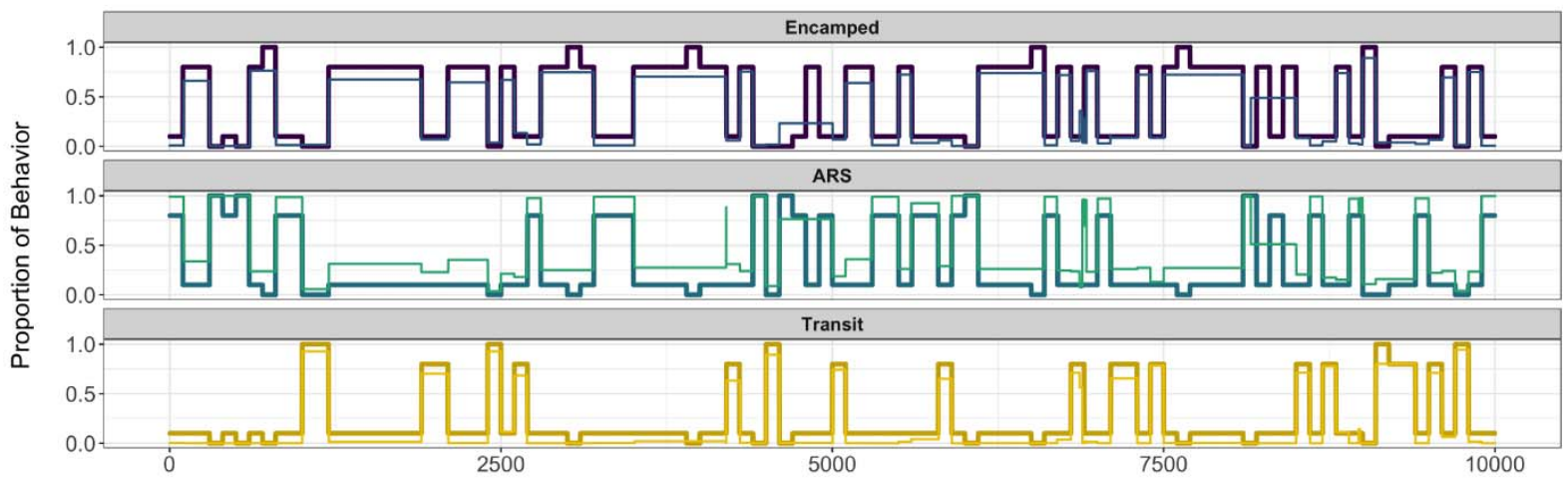

Figure 6 Evaluation of model performance for a single simulation, as an example, for (a) breakpoint estimation, (b)

determination of the shape of behavioral states, and (c) estimating behavior proportions over time. (a) A time series of true 
behavior are shown; thick, dark lines indicate true behavior proportions whereas thin, light lines indicate estimated proportions

494 of behaviors.

The accuracy of the estimated step lengths and turning angles distributions were

497 relatively consistent across each set of simulations. For tracks generated from uncommon

498 distributions, M4 was slightly more accurate than the HMM, but much more accurate than

499 EMbC and segclust2d across all track lengths (Appendix S9). However, HMM estimates were

500 slightly more accurate than the Bayesian model on tracks of all lengths when generated from

501 common distributions (Appendix S9). When viewed as continuous distributions, it is clear that

502 the HMM, EMbC, and segclust2d models had difficulty estimating the true distributions of step

503 lengths and turning angles regardless of track length for the simulation with uncommon

504 distributions (Appendix S10). On the other hand, the HMM was able to perfectly estimate the

505 state-dependent distributions of the simulations generated from common distributions (Appendix 506 S10).

\subsection{Snail kite analysis}

The segmentation of 26 snail kite trajectories using M4 took approximately 61 min to run

510 and estimated 1 to 64 breakpoints for these individuals. Breakpoints were then used to define 444

511 track segments from all individuals (Fig. 7a). These segments were clustered into states using

512 M4, which took approximately $27 \mathrm{~s}$ to run. It appeared that there were likely three behavioral

513 states, which comprised $91.6 \%$ of all state assignments on average (Fig. 7b). To ensure that these

514 three states were biologically interpretable, distributions of step lengths and turning angles were

515 also evaluated (Fig. 7c). The distributions showed: 1) a slow and tortuous behavior; 2) a tortuous 
bioRxiv preprint doi: https://doi.org/10.1101/2020.11.05.369702; this version posted June 9, 2021. The copyright holder for this preprint (which was not certified by peer review) is the author/funder, who has granted bioRxiv a license to display the preprint in perpetuity. It is made available under aCC-BY 4.0 International license.

516 behavior with intermediate speed; and 3) a fast and directed behavior. For this reason, these

517 behaviors were labelled 'encamped, 'ARS', and 'transit', respectively.

(a)
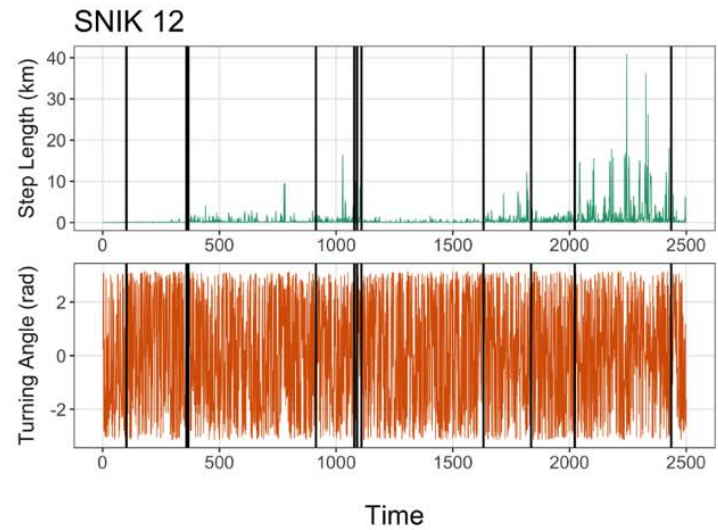

(b)

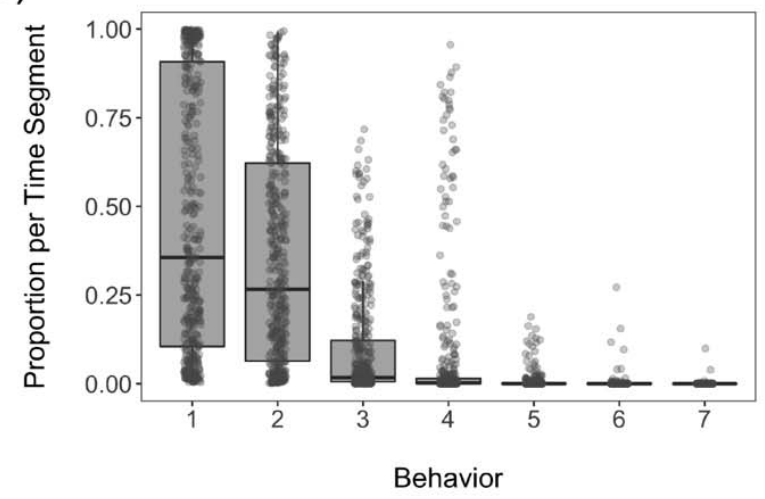

(C)

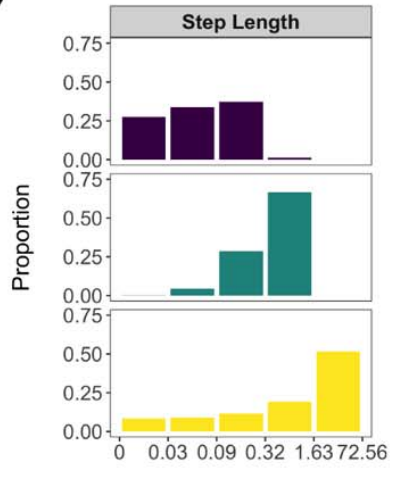

Bin

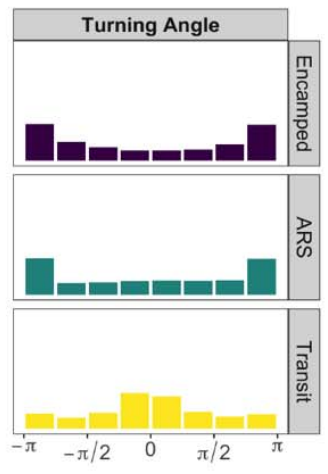

Bin

519 Figure 7 Snail kite results from track segmentation and determination of the most likely number of behavioral states. (a) A

520 subset of a time series from a single individual (SNIK 12) that displays estimated breakpoints (black lines) overlaying each data

521 stream. Time series of step lengths and turning angles are shown on a continuous scale in relation to estimated breakpoints for 
ease of interpretation. (b) Boxplot showing the estimated proportions for each of the seven possible behaviors from all 444 track

523 segments analyzed. (c) Distributions of step lengths $(\mathrm{km})$ and turning angles (rad) from each of the three retained behavioral

524 states, ordered from slowest to fastest.

525 Some individuals were only tracked for a short period of time and did not leave the natal

526 area. However, 17 birds did emigrate from their natal site. Dispersal events were typically

527 denoted by a brief period of ARS or transit behavior (Figs. 8a, b, Appendix S9). The three

528 longest tracks, which belonged to snail kites tracked for more than a year (SNIK 12, SNIK 14,

529 and SNIK 15), displayed relatively synchronous behavior before, during, and after their first

530 breeding season. Two brief periods of high activity behavior that occurred during and

531 immediately following peak breeding season in 2019 may potentially represent pre- and post-

532 breeding dispersal events (Figs. 8a, c, d). 


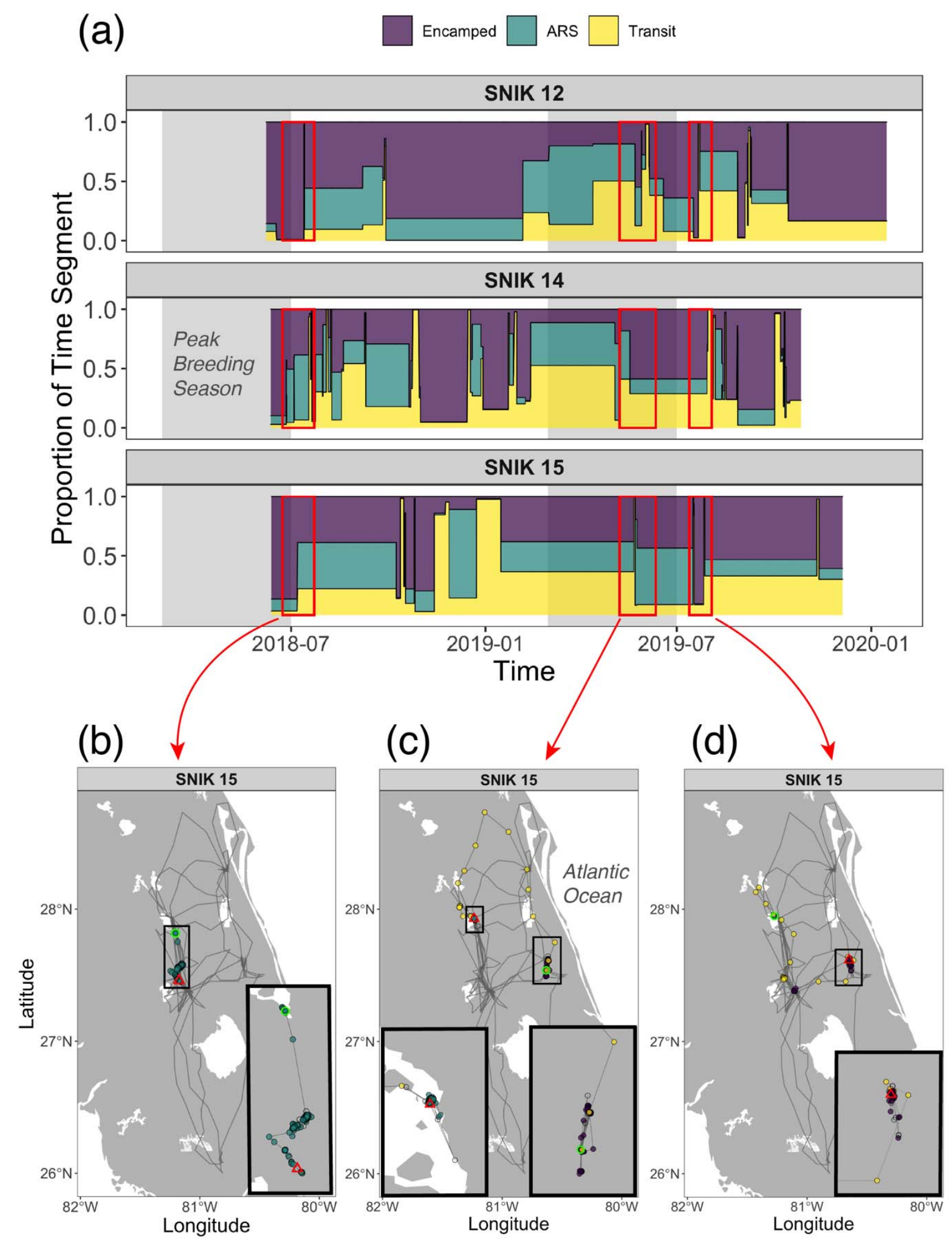


Figure 8 Patterns of movement behavior are shown over time for the three snail kites that were tagged over the longest

535 durations, with particular focus on SNIK 15. (a) Time series of behavior proportions are shown with respect to peak breeding

536 season (grey panels) for each of the three snail kites, with possible natal, pre-breeding, and post-breeding dispersal events

537 denoted by red boxes. Maps of SNIK 15 movement depict the starting (green circle) and ending locations (red triangle) for (b)

538 natal, (c) pre-breeding, and (d) post-breeding season dispersal events, as well as the dominant behavior associated with each

539 track segment. Observations without behavior estimates (i.e., observations not recorded at 1-h time intervals) are shown as

540 open points.

\section{Discussion}

We demonstrated that our Bayesian M4 framework (available within the bayesmove R

544 package) can accurately identify changes in behavioral states, reliably estimate the most likely

545 number of behavioral states, and properly characterize the state-dependent distributions of data

546 streams. This two-stage model treats track segments as the unit of interest (as opposed to

547 observations) and relies on the discretization of data streams to avoid the need to specify

548 parametric probability distributions. Importantly, the proposed method is relatively

549 computationally efficient, a key characteristic given the ever-increasing storage capacities of

550 modern sensors and their ability to measure a growing number of intrinsic and environmental

551 variables (Whitford and Klimley 2019; Williams et al. 2020). A comparison of model

552 performance in addition to the analysis of an empirical dataset highlight the utility of the M4

553 framework.

555 4.1 Method Comparison

Although BCPA was notably faster at track segmentation than M4, accuracy of the

557 estimated breakpoints was much higher in the latter. Additionally, M4 was faster and exhibited 558 greater accuracy of breakpoint estimates than the segclust $2 \mathrm{~d}$ method, which was not able to 
559 successfully analyze simulated tracks of 50000 observations. Since the accuracy of the

560 segclust2d method was not much greater than the BCPA for either set of simulations (Figs. 4b,

$5615 b)$, it appears that BCPA's reliance on a single derived variable (i.e., persistence velocity)

562 instead of separate data streams was not as limiting as was initially expected.

564 and account for cyclical patterns (Patterson et al. 2017; McClintock and Michelot 2018), they

565 can also be restrictive in some of their assumptions. Standard forms of HMMs require the use of

566 parametric distributions, which may not fit the data streams well (Appendix S9; Langrock et al.

567 2018). While HMMs displayed better performance than M4 when the selected parametric

568 distributions matched the true underlying distributions (Fig. 5c), we find that the proposed

569 methodology performed better than HMMs when the selected parametric distributions did not

570 match the true underlying distribution. By comparison, the segclust $2 \mathrm{~d}$ and EMbC methods are

571 straightforward to apply when estimating latent behavioral states from a set of tracks, but appear

572 limited by their assumption of Gaussian distributions when partitioning observations into

573 segments or into states, respectively. Since the most common data streams (i.e., step lengths and

574 turning angles) are not typically modeled with a Gaussian distribution (McClintock et al. 2020),

575 this likely contributes to the lower accuracy of these models.

576 The determination of the most likely number of states is another issue when fitting

577 clustering models and HMMs since this is typically unknown a priori and is directly impacted by

578 how well the selected parametric distributions characterize the states (Pohle et al. 2017).

579 Unfortunately, HMMs often require multiple models to be fit and compared using information

580 theoretic approaches, which tend to favor a greater number of states than are truly present and

581 come at a high computational cost ( $\mathrm{Li}$ and Bolker 2017; Pohle et al. 2017). Importantly, while 
582 M4 allows for up to 7 behavioral states, we only attempted to fit HMMs with 2 to 4 behavioral

583 states. Even in this limited context, fitting HMMs was already much slower than fitting M4. Had

584 we attempted to fit HMMs with 2 to 7 behavioral states, the amount of time required for this

585 would be substantially larger than what we report in Figs. 4 and 5. A similar issue is present in

586 segclust $2 \mathrm{~d}$, where models are fit with every possible number of track segments and states before

587 comparing via BIC. A different problem is posed by the EMbC model, which imposes four states

588 by default when analyzing step lengths and turning angles. These issues are directly addressed by

589 our framework since we use a mixed-membership model (LDA) with a penalizing prior to cluster

590 track segments, enabling the estimation of the most likely number of states and the state-

591 dependent distributions in a single step. While existing methods can provide useful behavioral

592 inference depending upon the ecological question and dataset, the M4 framework provides a

593 powerful alternative when behaviors are complex, multiple data streams are available and these

594 data are not well characterized by parametric distributions, and/or datasets are large.

595

$596 \quad 4.2$ Empirical applications

597 Three behavioral states were clearly estimated for the snail kite dataset, which was

598 supported by biologically relevant distributions of step lengths and turning angles. The

599 'encamped' state likely represents fine-scale behaviors that include resting, feeding, and time

600 spent at the nest (as a fledgling or reproductive adult). On the other hand, the 'ARS' state likely

601 includes exploration for nearby suitable habitat as well as foraging bouts (Martin et al. 2006; Pias

602 et al. 2016). Finally, the 'transit' state includes fast, directed movements associated with

603 dispersal of snail kites in addition to departure from wetlands experiencing low water levels

604 (Robertson et al. 2017). 
The time series of snail kite behavior proportions showed variability in the timing of

606 emigration from natal sites among individuals, but changes in behavior were generally

607 synchronous in the three birds that reached maturity. This variability in the timing of emigration

608 from natal sites could be due to a variety of factors, such as hatching date, body condition, and

609 local environmental conditions (Rodgers and Schwikert 2003; Fletcher et al. 2015; Cattau et al.

610 2016). The shifts in behavior proportions appeared to show multiple phases of high and low

611 activity, some of which seem to match the phenology of natal dispersal (summer), pre-breeding

612 dispersal (early spring), and post-breeding dispersal (late summer) (Bennetts and Kitchens 2000).

613 While the continued monitoring of these tagged birds should provide greater evidence for the

614 characterization of activity budgets over ontogeny, future research could also explore the

615 primary drivers of snail kite movement and habitat use within each behavioral state through the

616 inclusion of environmental covariates.

\subsection{Caveats and extensions}

In addition to the M4 method proposed by this study, other non-parametric state

620 estimation methods have been previously developed (Nams 2014; Sur et al. 2014; Langrock et al.

621 2018). In one such example, the behavioral movement segmentation (BMS) model proposed by

622 Nams (2014) uses a combination of direct search optimization, iterative sampling, and k-means

623 clustering to estimate latent states from track segments. BMS is similar to our proposed M4

624 framework in that both methods are non-parametric, partition multiple data streams into

625 segments, and cluster segments into latent states (Nams 2014). However, M4 differs both

626 technically and conceptually from BMS in that M4 proposes breakpoints using RJMCMC, the

627 number of likely states are estimated within a single model run (instead of using multi-model 
628 selection), and track segments are expected to be comprised of multiple states rather than just

629 one. We believe that practitioners should carefully evaluate the properties and assumptions of

630 different methods to determine the best method to properly analyze their data and address their

631 objectives.

Although M4 effectively classified behavioral states from both simulated and empirical

633 tracks, there are some limitations to this approach. The selection of the number and width of bins

634 when discretizing data streams is a subjective choice that impacts the results from the

635 segmentation model and ultimately the estimation of behavioral states. Additionally, our model

636 implicitly assumes that location error is negligible or requires that it be accounted for via another

637 method. Although our model can analyze data streams from regular or irregular time intervals,

638 this will also depend on the inherent properties of the data streams themselves. Since step lengths

639 and turning angles are calculated from multiple successive observations, these values will not be

640 comparable once the data are not close to a regular time interval. However, variables such as net

641 squared displacement (the squared distance from the starting location to all other relocations) can

642 be analyzed over irregular time intervals.

643 M4 can be extended to analyze other types of data streams and can include prior

644 knowledge on the timing of behavioral shifts. Although only step lengths and turning angles

645 were analyzed for the simulated and empirical tracks, additional ancillary data coming from the

646 sensor (e.g., elevation, salinity, temperature, or accelerometer data) could be used to make

647 behavioral inference. These data streams could come from all types of distributions (i.e.,

648 continuous, discrete, bounded between 0 and 1). It is also relatively straightforward to deal with

649 zero-inflated data by including all zeroes in a single bin. Additionally, our segmentation model

650 can be implemented in a semi-supervised fashion, by which practitioners pre-specify breakpoints 
651 for the time series based on a priori knowledge and these breakpoints will be considered by the

652 RJMCMC algorithm. This may be particularly useful if daily activity patterns are expected or if

653 only one of several possible states can be clearly identified.

\section{Acknowledgments}

This work was supported in part by the University of Florida Biodiversity Institute

657 (UFBI), the US Department of Agriculture's National Institute of Food and Agriculture (USDA-

658 NIFA) McIntire-Stennis project 1005163, the US National Science Foundation (NSF) award

6591458034 and 2040819, the Florida Fish and Wildlife Conservation Commission (FWC), and by

660 the US Army Corp of Engineers (USACE). The tagging of snail kites was approved and

661 conducted under UF IACUC no. 201708334. JAC was supported by a postdoctoral fellowship

662 from the University of Florida Informatics Institute (UFII) Fellowship Program. We would like

663 to thank Tyler Beck for his assistance with field work and Brenda Betancourt for her feedback

664 during model and package development. Additionally, we are grateful to Rocío Joo and Mathieu

665 Basille for their valuable comments on an earlier draft of this paper.

666

667 Authors' Contributions

668 J.A.C. and D.V. conceived the ideas and designed methodology; C.L.P. and R.J.F.

669 collected the empirical data; J.A.C., C.L.P., and D.V. analyzed the data; J.A.C. and D.V. led the

670 writing of the manuscript. All authors contributed critically to the drafts and gave final approval

671 for publication.

672

673 Data Availability 
674 All code mentioned here for the Bayesian framework is available within the bayesmove

675 package for R hosted on CRAN at https://CRAN.R-project.org/package=bayesmove. The

676 development version of the package is available on GitHub at

677 https://github.com/joshcullen/bayesmove and a full set of vignettes can be found at

678 https://joshcullen.github.io/bayesmove. The code to generate the simulations and perform

679 method comparison are available on Zenodo (https://doi.org/10.5281/zenodo.4898208). The

680 Everglade snail kite telemetry data have not been made available since this is a federally listed

681 endangered species and the location data are sensitive.

682

\section{References}

684 Bennetts RE, Kitchens WM. 2000. Factors influencing movement probabilities of a nomadic

685 food specialist: proximate foraging benefits or ultimate gains from exploration? Oikos

686 91:459-467. doi:10.1034/j.1600-0706.2000.910306.x.

687 Burnham KP, Anderson DR. 2002. Model selection and multimodel inference: A practical

688 information-theoretic approach. 2nd ed. New York: Springer.

689 Cattau CE, Fletcher RJ, Reichert BE, Kitchens WM. 2016. Counteracting effects of a non-native

690 prey on the demography of a native predator culminate in positive population growth.

691 Ecological Applications 26:1952-1968. doi:10.1890/15-1020.1/suppinfo.

692 Cullen JA, Valle D. 2021. bayesmove: Non-Parametric Bayesian Analysis of Animal Movement.

$693 \quad \mathrm{R}$ Package version 0.2.0.

694 Denison DGT, Holmes CC, Mallick BK, Smith AFM. 2002. Bayesian methods for nonlinear

695 classification and regression. Chichester, UK: John Wiley \& Sons.

696 Edelhoff H, Signer J, Balkenhol N. 2016. Path segmentation for beginners: an overview of 
current methods for detecting changes in animal movement patterns. Movement Ecology 4:21. doi:10.1186/s40462-016-0086-5.

Fletcher RJ, Robertson EP, Wilcox RC, Reichert BE, Austin JD, Kitchens WM. 2015. Affinity for natal environments by dispersers impacts reproduction and explains geographical structure of a highly mobile bird. Proceedings of the Royal Society B: Biological Sciences 282:20151545. doi:10.1098/rspb.2015.1545.

Fraser KC, Davies KTA, Davy CM, Ford AT, Flockhart DTT, Martins EG. 2018. Tracking the conservation promise of movement ecology. Frontiers in Ecology and Evolution 6:150. doi:10.3389/fevo.2018.00150.

Garriga J, Palmer JRB, Oltra A, Bartumeus F. 2016. Expectation-maximization binary clustering for behavioural annotation. PLoS ONE 11:1-26. doi:10.1371/journal.pone.0151984. Clustering. R package version 2.0.3.

Green PJ. 1995. Reversible jump Markov chain Monte Carlo computation and Bayesian model determination. Biometrika 82:711-732. doi:10.2307/2337340.

712 Gurarie E. 2014. bcpa: Behavioral change point analysis of animal movement. R package version

$$
1.1 .
$$

714 Gurarie E, Andrews RD, Laidre KL. 2009. A novel method for identifying behavioural changes in animal movement data. Ecology Letters 12:395-408. doi:10.1111/j.1461-

717 Gurarie E, Bracis C, Delgado M, Meckley TD, Kojola I, Wagner CM. 2016. What is the animal 718 doing? Tools for exploring behavioural structure in animal movements. Journal of Animal 719 Ecology 85:69-84. doi:10.1111/1365-2656.12379. 
720

721

722

723

724

725

726

727

728

729

730

731

732

733

734

735

736

737

738

739

740

741

Hudon SF, Zaiats A, Roser A, Roopsind A, Barber C, Robb BC, Pendleton BA, Camp MJ, Clark PE, Davidson MM, et al. 2021. Unifying community detection across scales from genomes to landscapes. Oikos. doi:10.1111/oik.08393.

Hussey NE, Kessel ST, Aarestrup K, Cooke SJ, Cowley PD, Fisk AT, Harcourt RG, Holland KN, Iverson SJ, Kocik JF, et al. 2015. Aquatic animal telemetry: A panoramic window into the underwater world. Science 348:1255642. doi:10.1126/science.1255642.

John GH, Langley P. 1995. Estimating continuous distributions in Bayesian classifiers. In: Proceedings of the Eleventh Conference on Uncertainty in Artificial Intelligence. Morgan Kaufmann Publishers Inc. p. 338-345.

Jonsen I. 2016. Joint estimation over multiple individuals improves behavioural state inference from animal movement data. Scientific Reports 6:20625. doi:10.1038/srep20625.

Jonsen ID, McMahon CR, Patterson TA, Auger-Méthé M, Harcourt R, Hindell MA, Bestley S. 2019. Movement responses to environment: fast inference of variation among southern elephant seals with a mixed effects model. Ecology 100:e02566. doi:10.1002/ecy.2566.

Joo R, Boone ME, Clay TA, Patrick SC, Clusella-Trullas S, Basille M. 2020. Navigating through the r packages for movement. Journal of Animal Ecology 89:248-267. doi:10.1111/13652656.13116.

Joo R, Picardi S, Boone ME, Clay TA, Patrick SC, Romero-Romero VS, Basille M. 2020. A decade of movement ecology. arXiv preprint:2006.00110.

Kays R, Crofoot MC, Jetz W, Wikelski M. 2015. Terrestrial animal tracking as an eye on life and planet. Science 348:aaa2478. doi:10.1126/science.aaa2478.

Kitagawa G. 1987. Non-gaussian state-space modeling of nonstationary time series. Journal of the American Statistical Association 82:1032-1041. doi:10.1080/01621459.1987.10478534. 
743 Langrock R, Adam T, Leos-Barajas V, Mews S, Miller DL, Papastamatiou YP. 2018. Spline-

744 based nonparametric inference in general state-switching models. Statistica Neerlandica

745 72:179-200. doi:10.1111/stan.12133.

746 Li M, Bolker BM. 2017. Incorporating periodic variability in hidden Markov models for animal 747 movement. Movement Ecology 5:1. doi:10.1186/s40462-016-0093-6.

748 Martin J, Nichols JD, Kitchens WM, Hines JE. 2006. Multiscale patterns of movement in 749 fragmented landscapes and consequences on demography of the snail kite in Florida. :527539. doi:10.1111/j.1365-2656.2006.01073.x.

751 McClintock BT, Langrock R, Gimenez O, Cam E, Borchers DL, Glennie R, Patterson TA. 2020.

752 Uncovering ecological state dynamics with hidden Markov models. Ecology Letters.

753 doi:10.1111/ele.13610.

754 McClintock BT, Michelot T. 2018. momentuHMM: R package for generalized hidden Markov

755 models of animal movement. Methods in Ecology and Evolution 9:1518-1530.

756 doi:10.1111/2041-210X.12995.

757 Michelot T, Langrock R, Patterson TA. 2016. moveHMM: an R package for the statistical

758 modelling of animal movement data using hidden Markov models. Methods in Ecology and

759 Evolution 7:1308-1315. doi:10.1111/2041-210X.12578.

760 Nams VO. 2014. Combining animal movements and behavioural data to detect behavioural $761 \quad$ states. Ecology Letters 17:1228-1237. doi:10.1111/ele.12328.

762 Nathan R, Getz WM, Holyoak M, Kadmon R, Saltz D, Smouse PE. 2008. A movement ecology 763 paradigm for unifying organismal movement research. Proceedings of the National 764 Academy of Sciences 105:19052-19059. doi:10.1021/i360006a005.

765 Patin R, Etienne M-P, Lebarbier E, Benhamou S. 2019. segclust2d: Bivariate 
767 Patin R, Etienne MP, Lebarbier E, Chamaillé-Jammes S, Benhamou S. 2020. Identifying

768 stationary phases in multivariate time series for highlighting behavioural modes and home range settlements. Journal of Animal Ecology 89:44-56. doi:10.1111/1365-2656.13105. practical challenges. AStA Advances in Statistical Analysis 101:399-438.

774 Pias KE, Fletcher RJ, Kitchens WM. 2016. Assessing the value of novel habitats to snail kites through foraging behavior and nest survival. Journal of Fish and Wildlife Management

Pohle J, Langrock R, van Beest FM, Schmidt NM. 2017. Selecting the number of states in hidden Markov models: Pragmatic solutions illustrated using animal movement. Journal of

781 Potts JR, Börger L, Scantlebury DM, Bennett NC, Alagaili A, Wilson RP. 2018. Finding turning782 points in ultra-high-resolution animal movement data. Methods in Ecology and Evolution Agricultural, Biological, and Environmental Statistics 22:270-293. doi:10.1007/s13253-

784 Reichert B, Cattau C, Fletcher Jr. R, Sykes Jr. P, Rodgers Jr. J, Bennetts R. 2020. Snail Kite USA: Cornell Lab of Ornithology. 
790 Rodgers JA, Schwikert ST. 2003. Breeding chronology of snail kites (Rostrhamus sociabilis

791 plumbeus) in central and south Florida wetlands. Southeastern Naturalist 2:293-300. doi:10.1656/1528-7092(2003)002[0293:bcoskr]2.0.co;2.

793 Sur M, Skidmore AK, Exo KM, Wang T, Ens BJ, Toxopeus AG. 2014. Change detection in animal movement using discrete wavelet analysis. Ecological Informatics 20:47-57. doi:10.1016/j.ecoinf.2014.01.007.

Valle D, Baiser B, Woodall CW, Chazdon R. 2014. Decomposing biodiversity data using the Latent Dirichlet Allocation model, a probabilistic multivariate statistical method. Ecology movement strategies revealed through novel clustering of emergent movement patterns. Scientific Reports 7:44052. doi:10.1038/srep44052.

Whitford M, Klimley AP. 2019. An overview of behavioral, physiological, and environmental sensors used in animal biotelemetry and biologging studies. Animal Biotelemetry 7:26. doi:10.1186/s40317-019-0189-z. HM, Franconi N, Gómez-Laich A, et al. 2020. Optimizing the use of biologgers for 